Vol. 50 (1994) [135-165]

\title{
ANALYSIS ON SUPERSPACE: AN OVERVIEW
}

\author{
Vladimir G. Pestov
}

\begin{abstract}
The concept of superspace is fundamental for some recent physical theories, notably supersymmetry, and a mathematical feedback for it is provided by superanalysis and supergeometry. We survey the state of affairs in superanalysis, shifting our attention from supermanifold theory to "plain" superspaces. The two principal existing approaches to superspaces are sketched and links between them discussed. We examine a problem by Manin of representing even geometry (analysis) as a collective effect in infinite-dimensional purely odd geometry (analysis), by applying the technique of nonstandard (infinitesimal) analysis.
\end{abstract}

\section{FOREWORD}

"Superspace is the greatest invention since the wheel" (see [59]). I don't know how many of us would agree with this radical statement, and it was only intended to be a jocular epigraph, of course. However, the concept of superspace is thriving indeed in theoretical physics of our days. Under superspace physicists understand our space-time labeled not only with usual coordinates $\left(x^{\mu}\right)$, but also with anticommuting coordinates $\left(\theta^{\alpha}\right)$, and endowed with a representation of a certain group or, better still, supergroup, whatever it means. The anticommuting coordinates represent certain internal degrees of freedom of the physical system. This concept was actually known in physics since the early 60 s or late 50 s $[138,138,91,28]$, but what made it so important was the discovery of supersymmetry $[144,131,145]$, which is an essentially new form of symmetry unifying fermions (particles of matter) with bosons (particles carrying interaction between bosons). One should also acknowledge the stimulating rôle of supergravity [97].

The prefix "super-", which originally merely reflected the fact that superspace is an extension of spacetime incorporating anticommuting (odd, fermionic) coordinates, has spread all over mathematical theories dealing with superspace, and has acquired a tint

Received 8th November, 1993

An extended version of the Invited Lecture at the Analysis Miniconference, The University of Wollongong, N.S.W., Australia, delivered on July 2, 1993. I am deeply indebted to the Organisers, especially Martin Bunder, Sidney Morris, Rod Nillsen, and Graham Williams, for inviting me to speak at the Miniconference and for their hospitality at Wollongong.

Copyright Clearance Centre, Inc. Serial-fee code: 0004-9729/94 \$A2.00+0.00. 
of pretention. It gave birth to such unhappy terms as superanalysis, supergeometry, superalgebra, and even supermathematics - terms, of which any pure mathematician can be hardly expected to approve. Felix Berezin, one of the creators of mathematics of superspace, has chosen for his book [24] the title "Introduction to Algebra and Analysis with Anticommuting Variables." (All the same, after his premature death the book was edited in the West under the title "Introduction to Superanalysis" [25].) The term Analysis on Superspace, accepted for the purposes of the present survey, is hopefully both adequate and neutral.

However, mathematics of superspace is not only of definite interest by itself, but also provides the simplest possible nontrivial example of a noncommutative extension of classical analysis and geometry, and this is an example where practically everything works well. In particular, one approach to quantum group theory simply generalises the theory of supergroups in a certain way, and from this viewpoint "supermathematics" is an invaluable testing ground for new concepts and results (see, for example, [90, Section 1.4]). Supermathematics looks almost "classical" against the newly emerging branches of noncommutative geometry - consider an example from [49], where Alain Connes reformulates a certain result on noncommutative spaces, in order to reveal its significance better, as a statement about the $(1,1)$-dimensional supergroup $\mathbb{R}^{(1,1)}$. However, even apart from that, there are mathematical questions of depth and significance about superspace that still remain open.

Analysis on superspace is, of course, too close to mathematical and theoretical physics in order not to benefit - and suffer at the same time - from the large number of articles on the subject belonging, entirely or in part, to speculative mathematics. (see the recent outstanding analysis of this phenomenon performed by Jaffe and Quinn [70].) We feel strongly that because of this there is a need to keep alive a constant stream of purely mathematical research in the area, if we wish to make the topic accepted after all as an integral part of mathematics. On the contrary, what actually happened a few years ago, was that after an outbreak of interest, supermathematics as such was almost deserted by researchers (some of whom moved to newly fashionable area of quantum groups).

\section{MOTIVATION: SUPERSYMMETRIC QUANTUM MECHANICS}

We shall sketch briefly and very loosely what physicists would call "a toy model" of SUSY. (For a presentation of SUSY in depth, the reader mathematician is referred to $[2,36,46,51,69,127]$ and especially $[59,50,146]$.)

In quantum mechanics, one has a Hilbert space $\mathcal{H}$, the state space, its elements being called states of system in question, and a number of self-adjoint densely defined operators on $\mathcal{H}$, called observables of the system. One of the most important observables 
is the Hamiltonian operator, $H$, the quantum analog of the energy of a system.

If a system contains both bosons and fermions, the state space $\mathcal{H}$ is split into the orthogonal sum $\mathcal{H}_{0} \oplus \mathcal{H}_{1}$, where $\mathcal{H}_{0}$ is the state space for bosons and $\mathcal{H}_{1}$ is the state space for fermions. In such case $\mathcal{H}$ is called a $\mathbb{Z}_{2}$-graded, or simply graded, Hilbert space. The underlying idea of supersymmetry is to introduce a new operator, $Q$, on $\mathcal{H}$, which interchanges bosons and fermions: $Q\left(\mathcal{H}_{0}\right) \subset \mathcal{H}_{1}, Q\left(\mathcal{H}_{1}\right) \subset \mathcal{H}_{0}$ (such an operator would be called an odd operator), commutes with the Hamiltonian $(Q H=H Q)$ and in addition is such that $Q^{2}=H$. In other words, one wishes to extract the odd (that is, mixing up bosonic and fermionic states) square root of the Hamiltonian operator, which would interchange bosons with fermions. The operator $Q$ is called a generator of supersymmetry, or a supercharge.

Let us consider a system with one bosonic and one fermionic degree of freedom. In the Schrödinger representation of such a system, $\mathcal{H}$ must be the space of functions $f$ depending on two coordinates, say $x$ and $\theta$. The position operator of the bosonic particle is of the form $f \mapsto x f$, and in the fermionic case it is $f \mapsto \theta f$. While the position, operators of bosonic particles commute with each other and fermions, the position operators of fermionic particles pairwise anticommute; in our simplest case, it leads to the condition $\theta^{2}=0$.

Let us now assume, by restricting the domain of definition, that our functions are differentiable. Then in the Taylor expansion for $f$ in $\theta$ all terms of the order $\geqslant 2$ vanish, and one has

$$
f(x, \theta)=f_{0}(x)+\theta f_{1}(x)+0
$$

(an example of what is called component analysis, or superfield expansion.) Here $f_{0}(x)$ and $f_{1}(x)$ are functions of a single variable $x$. The bosonic states are of the form $f(x)$, and the fermionic ones of the form $\theta f(x)$.

Introduce differentiation by $\theta$ by the rule $\frac{\theta \theta}{\partial \theta}=1$ plus $\mathbb{C}$-linearity. In particular, the second derivative in $\theta$ always vanishes. Extend differentiation by $x$ over all functions by making it linear with respect to $\theta$.

For simplicity, let us extract the odd square root from the momentum operator $\partial / \partial x$ instead of the Hamiltonian. Put

$$
Q=\theta \frac{\partial}{\partial x}+\frac{\partial}{\partial \theta}
$$

Then clearly

$$
\begin{aligned}
Q(f(x)) & =\theta \frac{\partial f(x)}{\partial x} \in \mathcal{H}_{1} \\
Q(\theta f(x)) & =f(x) \in \mathcal{H}_{0}
\end{aligned}
$$


so $Q$ is odd, and finally

as required.

$$
\begin{aligned}
Q^{2}(f(x)) & =Q\left(\theta \frac{\partial f(x)}{\partial x}\right)=\frac{\partial f(x)}{\partial x} \\
Q^{2}(\theta f(x)) & =Q(f(x))=\theta \frac{\partial f(x)}{\partial x}=\frac{\theta \partial f(x)}{\partial x}
\end{aligned}
$$

One can introduce integration in $\theta$ by letting

$$
\int d \theta=0, \quad \int \theta d \theta=1 .
$$

The integral above (called the Berezin integral) is extended over all functions $f=$ $f(x, \theta)$ by linearity. If $f(x)$ is an ordinary function in $x$, then it can be expanded in a terminating Taylor series

$$
f(x)=f(0)+\theta f^{\prime}(0)+0
$$

and therefore

$$
\int f(x) d \theta=\int\left[f(0)+\theta f^{\prime}(0)\right] d \theta=f^{\prime}(0)
$$

which fact is sometimes expressed by not quite precise statement that in superspace "differentiation is equivalent to integration."

Supersymmetry with a single generator $Q$ is called simple supersymmetry; if there is more than one "supercharge," or "generator of supersymmetry," $Q_{1}, \ldots, Q_{N}$, then it is called extended supersymmetry. It is easy to verify that the supersymmetry generators commute with (the generators of) the usual space-time translations and rotations, but anticommute among themselves:

$$
Q_{i} Q_{j}+Q_{j} Q_{i}=0 \text {. }
$$

One more puzzle: traditionally, it is known that the infinitesimal symmetries of a system form a Lie algebra; clearly, it is not the case with supersymmetry algebra, algebraically generated by translations, rotations, and supercharges.

This example reflects all essential features of superspace. It would be called a superspace of dimension $(1,1)$, that is, it is one-dimensional both in the even (bosonic) and the odd (fermionic) sectors. (Of course, complicated supersymmetric systems of field theory or string theory have infinitely many degrees of freedom.) The goal for a mathematician is: to provide a background for functions depending on odd (anticommuting) coordinates $\theta$; for their derivatives by such coordinates; for symmetry generators satisfying the above relations, et cetera.

The following heuristic sign, or Quillen, rule has proved extremely important throughout supermathematics: if an entity of parity $i$ moves past another entity of parity $j$, the factor of $(-1)^{i j}$ appears. (See, however, a warning in [88] against turning the sign rule into a metatheorem.) 


\section{Graded-COMmUtative algebras}

A graded algebra $\Lambda$ is an algebra over the basic field $\mathbb{K}$ with a fixed vector space decomposition $\Lambda \cong \Lambda^{0} \oplus \Lambda^{1}$, where $\Lambda^{0}$ is called the even and $\Lambda^{1}$ the odd part (sector) of $\Lambda$, in such a way that the parity $\tilde{x}$ of any element $x \in \Lambda^{0} \cup \Lambda^{1}$, defined by letting $x \in \Lambda^{\tilde{x}}, \tilde{x} \in \mathbb{Z}_{2}$, meets the following restriction:

$$
\widetilde{x * y}=\widetilde{x}+\tilde{y}, \quad x, y \in \Lambda^{0} \cup \Lambda^{1} .
$$

A graded algebra $\Lambda$ is called graded commutative if it is associative, unital, and

$$
x y=(-1)^{\widetilde{x y}} y x, \quad x, y \in \Lambda^{0} \cup \Lambda^{1} .
$$

The central example of graded commutative algebra is the Grassmann algebra $\wedge(\Xi)$ over a set $\Xi$ of odd ("fermionic") generators. It is freely generated by $\Xi \cup\{1\}$ modulo associativity, unitality, and the anticommutation relations:

$$
\xi_{i} \xi_{j}+\xi_{j} \xi_{i}=\delta_{i j}, \quad \xi_{i}, \xi_{j} \in \Xi
$$

A $\mathbb{Z}_{2}$-grading is introduced on $\Lambda(\Xi)$ in a unique fashion by requiring that $\Xi \subset \Lambda^{1}$. Any element of $\wedge(\Xi)$ is a (terminating) polynomial in odd variables

$$
a_{0}+a_{1} \xi_{1}+\cdots+a_{n} \xi_{n}+a_{12} \xi_{1} \xi_{2}+\cdots+a_{123 \ldots n} \xi_{1} \xi_{2} \ldots \xi_{n} .
$$

We shall use superscripts to designate monomials as follows

$$
\xi^{\mu}=\xi_{\mu_{1}} \xi_{\mu_{2}} \ldots \xi_{\mu_{k}}, \quad \xi^{\natural}=1
$$

The structure of a finite-dimensional Grassmann algebra is exposed in great detail in $[24,25]$ and $[47]$.

\section{Calculus in a Grassmann algebra}

A Grassmann algebra can be viewed in two ways: either as the algebra of superfunctions, or the algebra of supernumbers. Both existing approaches to superanalysis originate here.

(a) If one considers $\wedge(\Xi)$ as the algebra of superfunctions depending on purely odd coordinates $\xi \in \Xi$ only (functions which are polynomial in those formal variables), one can introduce formal differentiation as a $\mathbb{K}$-linear operator such that

$$
\frac{\partial}{\partial \xi_{i}}\left(\xi_{i} \xi^{\mu}\right)=\xi^{\mu}
$$


if $i \notin \mu$. For example,

$$
\frac{\partial}{\partial \xi_{1}}\left(\xi_{2} \xi_{1}+\xi_{1}+\xi_{2}\right)=\frac{\partial}{\partial \xi_{1}}\left(-\xi_{1} \xi_{2}+\xi_{1}+\xi_{2}\right)=-\xi_{2}+1
$$

The operator $\partial / \partial \theta$ satisfies the graded version of the Leibniz rule:

$$
D(f g)=D f \cdot g+(-1)^{\tilde{D} \tilde{f}} f \cdot D g
$$

In our case the parity of the differential operator $D=\partial / \partial \theta$ is 1 .

Invariance considerations make it necessary to define the integral (called the Berezin integral) as

$$
\begin{aligned}
\int_{B e r} d \xi_{i} & =0 \\
\int_{B e r} \xi_{i} d \xi_{i} & =1
\end{aligned}
$$

Though the integral was known for quite a while [53], it became a full-scale device after the concept of the superdeterminant (explaining how change of variables is performed) was introduced, independently in [4] and [24]. (See the classical reference [53] for explanations.) We do not touch upon the superdeterminant in this paper.

If $|\Xi|=n$, then one can think of $\wedge(\Xi)$ as the algebra of (super) functions on a superspace of dimension $(0, n)$. The calculus on this space is apparently completely understood, because of being purely formal.

Now one can supplement the algebra of functions with all ordinary ("even") functions, by tensoring $\wedge(\Xi)$ with the algebra of (say, smooth) functions on a domain $U \subseteq \mathbb{K}^{m}$ of dimension $m$. The resulting (algebraic) tensor product algebra

$$
C^{\infty}\left(U^{m, n}\right)=C^{\infty}(U) \otimes \wedge(\Xi)
$$

is called the algebra of (smooth) superfunctions on a coordinate superdomain $U$ of dimension $(m, n)$. For every $f=f(x, \xi)$ from this algebra, one has

$$
f(x, \xi)=f_{0}(x)+f_{1}(x) \xi_{1}+\cdots+f_{n}(x) \xi_{n}+f_{12}(x) \xi_{1} \xi_{2}+\cdots+f_{123 \ldots n}(x) \xi_{1} \xi_{2} \ldots \xi_{n}
$$

This expression is called superfield expansion of the superfunction $f$. One can easily extend the derivatives both by $x_{i}$ and $\xi_{j}$ over the algebra $C^{\infty}\left(U^{m, n}\right)$. The same is true for integration over $U$.

(b) If one treats elements of $\wedge(\Xi)$ as supernumbers, then there are clearly both even and odd supernumbers (as well as those of a mixed nature). Every element of the radical of $\wedge(\Xi)$ is nilpotent. Therefore, each supernumber $x$ spilts in a unique 
fashion as the sum of a number and a nilpotent. The number part of $x$ is denoted by $x_{B}$ and sometimes called the body part of $x$, and the nilpotent part, denoted by $x_{S}$, is sometimes called the soul part of $x$. The mapping $x \mapsto x_{B}$ - the body map - is, of course, well-known in mathematics under the name of the augmentation homomorphism (the only nontrivial character on the Grassmann algebra).

Any $C^{\infty}$ function $f: \mathbb{K} \rightarrow \mathbb{K}$ can be extended to a function $f^{\sim}: \wedge(\Xi)^{0} \rightarrow \wedge(\Xi)^{0}$ by applying the Taylor expansion:

$$
f^{\sim}(x)=\sum_{n=0}^{\infty} \frac{f^{(n)}\left(x_{B}\right)}{n !}\left(x_{S}\right)^{n} .
$$

Due to the fact that every element of $\Lambda(\Xi)^{0}$ is of the form "number + nilpotent," the above expansion terminates. This procedure is called soul expansion, or Grassmann analytic continuation. The same applies to the more general situation where a function maps smoothly $\mathbb{K}$ to $\wedge(\Xi)$. A smooth superfunction depending on even coordinates is just any mapping $f: \wedge(\Xi)^{0} \rightarrow \wedge(\Xi)$ extension of an ordinary smooth function $f: \mathbb{K} \rightarrow$ $\wedge(\Xi)$. A generic smooth superfunction of one even and one odd argument is a mapping $f: \wedge(\Xi) \rightarrow \wedge(\Xi)$ which admits a superfield expansion

$$
f^{\sim}(x, \xi)=f_{0}+f_{1} \xi
$$

where the component functions $f_{\alpha}: \wedge(\Xi)^{0} \rightarrow \wedge(\Xi)$ are smooth superfunctions depending on one even coordinate $x$. One can extend this definition in an obvious way to superfunctions depending on finitely many even and odd coordinates.

Clearly, to every superfunction in the sense of definition (a), there is a superfunction in the second sense (obtained by applying the Grassmann analytic continuation to all component functions), but not vice versa. Example: the constant superfunction

$$
f(x, \theta)=\xi_{1},
$$

where $\xi_{1}$ is a fixed odd generator of $\wedge(\Xi) \backslash \mathbb{K}$, is smooth in the sense of the second approach, but it is not obtained from any function in the sense of the first approach.

In other words, in (a) we have superfunctions without superconstants.

Everything above said makes sense for analytic functions as well.

\section{AN APPROACH TO SUPERSPACES INCORPORATING SUPERNUMBERS}

This approach may be, at a certain stretch, termed a "naïve" one. It was developed by Felix Berezin $[\mathbf{2 2}, \mathbf{2 4}]$ and Bryce DeWitt (as a chapter in his book [53]). (It is worth mentioning that Berezin worked with and greatly contributed to both approaches to 
supermanifolds, and arguably was completely satisfied with none, see the remarks of Leĭtes in [83].) Further developments are due to Alice Rogers [120, 121, 122, 123, 124, 125], Boyer and Gitler [35], Yvonne Choquet-Bruhat [44, 45], Vladimirov and Volovich [142, 143], Bruzzo [36, 37, 38], and others. This approach is somewhat easier to accept psychologically. Now we shall discuss it briefly, just by elaborating and extending the approach (b) from the previous Section.

A point of departure is a fixed graded-commutative algebra of supernumbers, or superconstants, $\Lambda$, called also the ground algebra of the theory. It is inconvenient to use finite-dimensional Grassmann algebras in this capacity, because any such algebra has the "top element", $\xi_{1} \xi_{2} \ldots \xi_{n}$, and therefore the annihilator of the nilpotent part is not reduced to zero. (For a long history of complications related to this feature of finite-dimensional Grassmann algebras, see $[35,128,129]$; it took some time to realise that the problem does indeed exist.) Because of that, one is in fact bound to start from a graded-commutative algebra with zero annihilator; any such algebra is infinite-dimensional, and in order to do analysis, one must endow it with a complete locally convex topology. Examples of such algebras are infinite-dimensional Grassmann algebras $\wedge(\Xi)$, endowed with a suitable topology (and completed thereafter). The $l_{1}$-type norm is a natural choice:

$$
\left\|\sum_{\mu \in M(\Xi)} a_{\mu} \xi^{\mu}\right\|:=\sum_{\mu \in M(\Xi)}\left|a_{\mu}\right|_{\mathbb{K}} .
$$

The completion of this algebra is denoted by $B_{\infty}$; it is a Banach graded commutative algebra, and every element is a sum number + quasinilpotent. It was introduced by Rogers $[120,122]$, and became popular because of many attractive properties [35, 36, $68,69,65,56]$. Other possibilities are: the DeWitt algebra

$$
\Lambda_{\infty}=\lim _{\longleftarrow} \wedge(n)
$$

which is a Fréchet algebra $[54,92,46]$; the algebra $\wedge(\Xi)$ with the strongest locally convex topology $[\mathbf{7 8}, \mathbf{1 2 5}]$, et cetera. (Rich collections of examples and, moreover, categorical constructions of such algebras can be found in $[77,102,103,106,110]$.)

It turns out that the choice of a ground algebra affects the analysis in a very essential way, and one cannot expect to obtain a wide range of results valid for any thinkable ground algebra $\Lambda$. Here is an instructive example. One can define the Grassmann analytic continuation of $C^{\infty}$ functions from an open subset $U \subset \mathbb{K}$ to the corresponding open subset $U^{\sim}=\beta^{-1} U$ of $B_{\infty}^{0}$ by the same rule as before

$$
f^{\sim}(x)=\sum_{n=0}^{\infty} \frac{f^{(n)}\left(x_{B}\right)}{n !}\left(x_{S}\right)^{n} .
$$


It is not surprising that the above series fails to converge for some functions $f$. Of course, it converges for $f$ analytic (because every element of the radical of a Banach algebra is quasinilpotent, and since $B_{\infty}$ is the direct sum of the number field and the radical, the complexification of this algebra possesses a well-defined holomorphic calculus). But if the Taylor coefficients of $f$ at some point $x \in U$ grow too fast (and it is a well-known fact that we can always make them grow as fast as desired), the series diverges in the Banach algebra $B_{\infty}$, in spite of quasi-nilpotency of $x_{S}$. This fact was observed in [64]. For some time the question asked in the above paper of describing those $C^{\infty}$ functions $f$ admitting analytic continuation to $B_{\infty}$ remained open, and the author's recent result [109] states that this is the case if and only if the Taylor series of $f$ actually converges in a neighbourhood of the point $x_{B}$ (such functions are called Pringsheim regular). A consequence of this result is the fact that every supersmooth function on $B_{\infty}$ must be Pringsheim regular, which excludes from consideration functions of the type $\exp \left(-1 / x^{2}\right), x \neq 0 ; 0, x=0$. This is, from our viewpoint, a disadvantageous complication of the theory, something extraneous, not in the true nature of superspace.

Next step is to form the linear superspace of dimension $m, n$ :

$$
\Lambda^{m, n}=\Lambda \otimes \mathbb{K}^{m, n} \equiv \underbrace{\Lambda^{0} \times \cdots \times \Lambda^{0}}_{m \text { times }} \times \underbrace{\Lambda^{1} \times \cdots \times \Lambda^{1}}_{n \text { times }} .
$$

A linear superspace, in other words, is the even part of a free $\Lambda$-module. A mapping $\Lambda^{m, n} \rightarrow \Lambda^{p, q}$ is called superlinear if it can be represented by means of an $(m+n) \times(p+q)$-matrix with entries from $\Lambda$. (For an elaborate treatment of matrix calculus and general aspects of linear superalgebra, see treatises $[\mathbf{8 8}, \mathbf{1 4}, \mathbf{2 5}, \mathbf{1 3 2}$, 133].) A superdifferentiable mapping $f$ at a point $x$ is defined as a Fréchet (or Gâteaux) differentiable mapping between locally convex spaces, such that the differential is a superlinear map. Now it is clear how to define the derivatives, supersmoothness of all orders, superanalyticity, et cetera. Of course, it is necessary to verify that such maps exist indeed and are abundant enough for a theory to be meaningful! (It seems, unfortunately, that such a need escaped the minds of many superanalysts, working in an infinite-dimensional context. See, for example, [76], where a version of superanalysis is developed in an enormously general context. It is beyond any doubt that for some ground algebras there are practically no smooth superfunctions other than polynomials. Consider an example of a "bad" locally convex graded-commutative algebra in our paper [101]; it turns out that superanalytic superfunctions in the sense of [76] may be even not $C^{1}$ smooth.) This is done with the help of Grassmann analytic continuation from $\mathbb{K}^{m}$ to $\Lambda^{m, 0}$, much in the same way as for the Grassmann algebra.

The next step would be to construct supermanifolds out of superdomains. A superdomain - within the present approach - is an open simply connected (or even con- 
tractible) subset of a linear superspace. The transition functions in an atlas are assumed to be supersmooth or superanalytic. This done, one defines the tangent module at a point as the $\Lambda$-module of graded derivations of germs of superfunctions. (A graded derivation of a graded $\Lambda$-algebra $A$ is a $\Lambda$-linear operator $d$ satisfying the graded Leibniz rule: $D(f g)=D(f) g+(-1)^{\widetilde{f D}} f D(g)$.) For reasonably good $\Lambda$, the tangent module is free, and its even part (the tangent linear superspace) is isomorphic to the model linear superspace. (In particular, one of the difficulties with finite-dimensional Grassmann algebras is that the tangent module is not free.) Super analogs of all the usual kinds of structures on manifolds, such as differential forms, connections, et cetera, are introduced and studied with greater or lesser success.

There is one subtle point of a topological nature. If one uses the usual tensor product topology on $\Lambda^{(m, n)}$ for patching the model superdomains together, one comes to the concept of Rogers supermanifold [120]. But there is another possibility. If the ground algebra $\Lambda$ is local and therefore every element admits a body and soul decomposition in a number part and a quasinilpotent part, then the body map $\beta: \Lambda \rightarrow \mathbb{K}$ (the usual augmentation homomorphism) is extended to the linear superspace $\Lambda^{m, n}$ by tensoring $\boldsymbol{\beta}$ with the projection $\mathbb{K}^{m, n} \rightarrow \mathbb{K}^{m}$. The DeWitt topology on $\Lambda^{m, n}$ is the pull-back of the standard topology on $\mathbb{K}^{m}$ via the body map $\beta$. If all coordinate domains of a supermanifold are DeWitt open and the transition functions commute with the body map, the supermanifold is called a DeWitt supermanifold [54, 45, 42]. (From the viewpoint of physical applications, it is not clear yet whether or not DeWitt supermanifolds suffice [43]. A mathematical relationship between the two concepts is discussed in $[115,116]$. It seems that in infinite dimensions Rogers supermanifolds become of importance; see [110] and a discussion below.) Topologically, a DeWitt supermanifold $M$ is (in the supersmooth case) a fibre bundle over a usual manifold, called the body of $M$. In the superanalytic case such a global section may not exist $[60]$.

\section{GeOMETRIC SUPERSPACES AND BeREZIN-LEITtes-Kostant SUPERMANIFOLdS}

This is a more sophisticated treatment, based upon sheaf theory and algebraic geometry. It is free of certain deficiencies of the "naive" construction.

Recall that a sheaf of graded-commutative algebras on a topological space $X$ is a collection of graded-commutative algebras $A(U)$ and their homomorphisms $r_{V}^{U}: A(U) \rightarrow$ $A(V)$ for all open subsets $U, V \subseteq X$ with $U \supseteq V$, satisfying a number of natural axioms. A topological space $X$ together with a sheaf of graded-commutative algebras on it, $\mathcal{O}_{X}$, is called a geometric superspace (or a locally ringed superspace [88]) if the stalks $\mathcal{O}_{X, x}$ of the structure sheaf are local algebras. This is a clear translation of the concept of a geometric space [52], basic for algebraic geometry, in the "super" 
language. A morphism $\phi$ between superspaces $X$ and $Y$ is a pair consisting of a continuous mapping $\phi_{0}: X \rightarrow Y$ and a sheaf morphism $\phi^{\sharp}: \mathcal{O}_{Y} \rightarrow \phi_{0, *}\left(\mathcal{O}_{X}\right)$ such that for every $x \in X$ the image of the maximal ideal $m_{x}$ of the stalk $\mathcal{O}_{Y, \phi(x)}$ under $\phi_{x}^{\sharp}$ is in $m_{\phi_{0}(x)}$.

For example, the (smooth) superdomain $U^{m, n}$ is a geometric superspace: the germs of superfunctions $f=f_{0}+f_{1} \xi_{1}+\cdots+f_{12 \ldots n} \xi_{1} \ldots \xi_{n}$ such that $f_{0}(x)=0$ form the maximal ideal in the corresponding stalk. (A detailed presentation of the structure theory of superdomains and calculus over them is to be found in [33].)

A supermanifold of dimension $(m, n)$ is a geometric superspace $X$ locally isomorphic to $C^{\infty}\left(U^{m, n}\right)$ (supersmooth case), or $C^{\omega}\left(U^{m, n}\right)$ (superanalytic case).

The theory is now developing very logically and quite on its own: tangent spaces, vector bundles, differential forms, connections et cetera., all sort of familiar geometric structures being "superized" and explored. For a good account, see any of the treatises $[7,14,25,79,82,83,88,134]$. One of the most complex tasks of the theory is to establish a consistent scheme of integration over supermanifolds (including the problem of integrating vector fields) and, naturally, supermanifold cohomology, see $[\mathbf{2 5}, \mathbf{2 9}, \mathbf{3 0}$, $38,41,46,54,63,76,80,83,85,88,96,114,124,135,143]$, and one can notice considerable difference of opinions and even a certain controversy about whether this task has been accomplished. We do not dwell on this topic any more. (Apparently even in the simplest case of a superspace of finite purely odd dimension there are still questions related to integral calculus deserving attention, especially in the context of cyclic cohomology [47].)

\section{LINKS BETWEEN THE TWO CONCEPTS}

Let $\Upsilon$ be a one-point superspace, that is, a pair $\Upsilon=\left(\star \Upsilon, \Lambda_{\Upsilon}\right)$ formed by a onepoint topological space and a graded-commutative local algebra (or, to be more precise, a constant sheaf of such algebras). Superspace morphisms from $\Upsilon$ to a geometric superspace $M$ are referred to as $\Upsilon$-points of $M$; we shall denote their totality by $p t_{\Upsilon} M$. For example, if $\mathbb{K}$ is the base field, $\mathbb{K}$-points of a superspace $M$ are the usual points, that is, elements of the underlying topological space $M_{0}$. The correspondence $M \mapsto p t_{\Upsilon} M$ is functorial, and it is called the functor of points from the category of supermanifolds and supermanifold morphisms to the category of sets and set-thenretic mappings. A superspace $M$ can be restored from the corresponding functor of points $\Upsilon \mapsto p t_{\Upsilon} M$ where the argument $\Upsilon$ runs over the totality of all one-point superspaces. (At least, this is so under certain additional conditions [105]; for instance, if $M$ is a supermanifold of finite dimension.)

To any section $f$ of the structure sheaf $\mathcal{S}_{M}$ over an open set $U \subset M_{0}$ (that is, a superfunction on $U$ ), there is associated a set-theoretic map $\Upsilon f: p t_{\Upsilon} U \rightarrow \Lambda_{\Upsilon}$, defined 
by letting $\Upsilon f(x):=x^{\sharp} f$ for all $x \in p t_{\Upsilon} U$. This way, superfunctions can be treated as usual functions defined on open subsets of wider sets than $M_{0}$. In the case $M$ is a "good" superspace (say, a smooth supermanifold), these extended sets of superpoints carry the structure of a manifold; moreover, they may be turned into supermanifolds over the algebra of superconstants $\Lambda_{\Upsilon}$. The resulting functor from the category of Berezin-Leites-Kostant supermanifolds to the category of supermanifolds over a ground algebra $\Lambda$ is called the functor of change of base. More generally, a similar functor connects the category of supermanifolds over a ground algebra $\Lambda$ to the category of supermanifolds over a ground algebra $\tilde{\Lambda}$ provided that $\Lambda \hookrightarrow \tilde{\Lambda}$. Of course, it is most important to bear in mind that the supermanifolds images of this functor do not exhaust all $\tilde{\Lambda}$-supermanifolds.

Denote by $\mathbb{K}^{0, q}$, or, for the sake of simplicity, just $q$, the superspace of the form $(\star, \wedge(q))$. It is a supermanifold (graded manifold) of dimension $(0, q)$. The q-points of a superspace $M$ are called q-points of $M$. It is implicitly assumed that any "good" superspace $M$ can be completely restored from its $q$-points.

The above concepts play a tremendous rôle in superspace theory, and they surfaced, either explicitly or implicitly, or in a different guise, in almost every solid investigation on the issue. Among the most important developments, let us mention those due to Batchelor [18, 19], Leĭtes and Bernstein [83, 31], DeWitt [54], and Schwarz [137]. An interesting discussion is to be found in the Appendix to [6]. It is, however, our overall impression that a unifying treatment of the topic is still missing.

\section{AXIOMS FOR SUPERMANIFOLDS}

In one attempt to merge the two approaches to supermanifold theory, Rothstein $[128,129]$ proposed a set of four axioms which any sensible category of supermanifolds should satisfy; later Bartocci, Bruzzo, Hernández Ruipérez and the author have shown $[15,16]$ that an additional axiom, calling for the completeness of the rings of sections of the structure sheaves, is necessary to characterise those Rothstein supermanifolds which are free from certain drawbacks.

Let $B$ denote a graded-commutative Banach algebra with unit. An $R$-superspace $(M, \mathcal{A}, e v)$ over $B$ is a triple formed by a paracompact topological space $M$, a sheaf $\mathcal{A}$ of graded-commutative $B$-algebras on $M$, and a morphism of sheaves of graded $B$-algebras $e v: \mathcal{A} \rightarrow \mathcal{C}_{M}, f \mapsto \tilde{f}=e v(f)$, called the 'evaluation morphism'; here $\mathcal{C}_{M}$ is the sheaf of continuous $B$-valued functions on $M$. An $R$-superspace is said to be local if the stalks $\mathcal{A}_{z}$ are local graded rings for any $z \in M$. A morphism of $R$-superspaces is a pair $\left(f, f^{\Downarrow}\right):\left(M, \mathcal{A}, e v^{M}\right) \rightarrow\left(N, \mathcal{B}, e v^{N}\right)$, where $f: M \rightarrow N$ is a continuous map and $f^{\sharp}: \mathcal{B} \rightarrow f_{*} \mathcal{A}$ is a morphism of sheaves of graded $B$-algebras, such that $e v^{M} \circ f^{\sharp}=f^{*} \circ e v^{N}$. 
One says that an $R$-superspace $(M, \mathcal{A}, e v)$ is an $(m, n)$ dimensional $R$-supermanifold if the following four axioms are satisfied.

AxIOM 1. $\operatorname{Der}^{*} \mathcal{A}$ (the dual sheaf of the sheaf of graded derivations of $\mathcal{A}$ ) is a locally free $\mathcal{A}$-module of rank $(m, n)$. Any $z \in M$ has an open neighbourhood $U$ with sections $x^{1}, \ldots, x^{m} \in \mathcal{A}(U)_{0}, y^{1}, \ldots, y^{n} \in \mathcal{A}(U)_{1}$ such that $\left\{d x^{1}, \ldots, d x^{m}, d y^{1}, \ldots, d y^{n}\right\}$ is a graded basis of $\operatorname{Der}^{*} \mathcal{A}(U)$.

The collection $\left(U,\left(x^{1}, \ldots, x^{m}, y^{1}, \ldots y^{n}\right)\right)$ is called a coordinate chart.

AxIOM 2. The mapping $\psi: U \rightarrow B^{m, n}, z \mapsto\left(\widetilde{x}^{1}(z), \ldots, \widetilde{x}^{m}(z), \widetilde{y}^{1}(z), \ldots, \widetilde{y}^{n}(z)\right)$ is a homeomorphism onto an open subset of $B^{m, n}$.

AxIOM $3^{\prime}$. For every $z \in M$ the ideal $\mathfrak{L}_{z}$ is finitely generated.

AXIOM 4. Let $\mathcal{D}(\mathcal{A})$ denote the sheaf of differential operators over $\mathcal{A}$, and let $f \in \mathcal{A}_{z}$, with $z \in M$. If $\widetilde{L(f)}=0$ for all $L \in \mathcal{D}(\mathcal{A})_{z}$, then $f=0$.

By imposing an additional axiom, calling for completeness of the algebras of sections in an appropriate locally convex topology, one can single out smooth supermanifolds, analytic supermanifolds, et cetera. All known examples (and even approaches) to supermanifolds fit into the above scheme if one enlarges the class of ground algebras $B$ to include complete locally convex graded-commutative algebras. Probably, the most reasonable additional condition to impose on them is to require any such $B$ to be topologically generated by the odd sector (the algebras of Grassmann origin, or GOalgebras, investigated under this or different names in $[102,103,106,109,16]$; they are exactly the completed Hausdorff quotients of locally convex Grassmann algebras).

For earlier attempts to merge the two approaches to a supermanifold, which now fit in the above axiomatic scheme, see $[8,9,10,11,12,13]$.

\section{INFINITE DIMENSIONAL SUPERSPACES}

Very little is known about these, although infinite-dimensional supermanifolds arise in mathematical physics (string theory, for one example [6]; gauge theories on supermanifolds $[1,40,62]$; conformal field theory [126], et cetera.); they are treated at an intuitive level. (A metaphysical discussion of this topic can be found in [6].) To realise how underdelevoped the infinite-dimensional theory still is, let us remark that (to the best of our knowledge) such a fundamental object as the supergroup of superdiffeomorphisms of a finite-dimensional smooth supermanifold has not been described yet as a genuine object of supergeometry, apart from the functor of points representation. (Though fragments of a relevant technique seem to exist already, see $[23,130]$.)

In the realm of the Berezin-Leites-Kostant approach, one can view a supermanifold of infinite dimension as a functor of points from the category $\mathcal{G}$ of (finite-dimensional or other) Grassmann algebras to the category of infinite-dimensional manifolds (modelled 
on Banach or more general locally convex spaces). A theory of this kind was deveioped by Molotkov [95] (in the Banach case). A disadvantage of such an approach is that these functors have no known representing objects. An attempt to construct such representing objects was made by Th. Schmitt [136] in the form of geometric superspaces with an additional structure, namely, the underlying topological spaces were locally convex manifolds (Schmitt worked in the holomorphic case), and in the algebras of sections special linear subspaces were distinguished. This approach - to our surprise - did not enjoy much popularity, perhaps because of its notable complexity.

The "naive" approach offers a possibility of carrying the finite-dimensional concepts straight to infinite-dimensions. Unfortunately, there are many subtleties, many traps to be avoided, and some of these were unnoticed by the authors. Since it is impossible to work over finite-dimensional Grassmann algebras of "supernumbers" without invoking either a functor of points or sheaves, one must fix an infinite-dimensional, thence topologised, Grassmann algebra, say $\Lambda$. Next comes the notion of a graded locally convex space, which in this approach must be a free graded locally convex module, that is, one starts from a genuine graded localy convex space $E=E^{0} \oplus E^{1}$ and constructs a free module $\Lambda \otimes E$, the tensor. product being topologised and completed. (Freeness is very essential as you develop analysis and geometry; for example, without freeness you would never be able to prove that the tangent space to a supermanifold is isomorphic to the model space). Now one encounters the choice of the right tensor product. For instance, one would expect that for two free graded LC modules, $E_{\Lambda}=\Lambda \otimes E$ and $F_{\Lambda}=\Lambda \otimes F$, the graded algebra of all endomorphisms $\mathcal{L}_{\Lambda}\left(E_{\Lambda}, F_{\Lambda}\right)$ is topologically free as a $\Lambda$-module (namely, an expected result would be that $\mathcal{L}_{\Lambda}\left(E_{\Lambda}, F_{\Lambda}\right) \equiv \Lambda \otimes \mathcal{L}(E, F)$ ). The author has shown [102] that if $\Lambda$ is an infinite-dimensional normed algebra then it is never so, if $\otimes$ means either projective or injective tensor product and $E=F=l_{1}$. As a matter of fact, only those ground algebras $\Lambda$ which are nuclear in the sense of Grothendieck can be used in the naive approach. This was overlooked, say, in [76].

However, there is one exception: if $\Lambda=\Lambda_{\infty}$, the DeWitt algebra inverse limit of finite-dimensional Grassmann algebras, then the resulting version of infinite-dimensional supermanifold looks satisfactory - at least, in some respects [110]. (From the classical viewpoint, it is a Fréchet manifold with an additional structure.)

We shall need infinite-dimensional BLK supermanifolds of a particular kind later: those of dimension $(0, \infty)$. Heuristically speaking, it is consistent with any of the existing approaches, to accept that the pair $(*, \Lambda)$, where $\Lambda$ is an infinite-dimensional Grassmann algebra (possibly topologised and completed after that) is such.

A discussion of a related open problem is to be found in our paper [107]. 


\section{RElative SUPERSPACES, OR, Where do THE GROUND Algebras COME FROM?}

The most disturbing thing about using "supernumbers" is the fact that their origin is completely unclear, and the choice for the "algebra of supernumbers" is totally arbitrary. The BLK approach is more elegant, and in addition it allows one to use methods from other areas of mathematics. At the same time, there is some evidence that by restricting ourselves to the BKL approach, we can lose some attractive properties. How to unify these two approaches?

The existing idea is to consider supermanifolds over supernumbers as objects in the relative category, that is, superfibre bundles of BLK supermanifolds with the base of the form Spec $\Lambda$. In other terms, they would become families of supermanifolds, indexed with elements of another supermanifold. What is now viewed as supernumbers, becomes just functions on the indexing supermanifold.

Let $\Lambda$ be a "supernumber algebra" (a graded-commutative algebra of Grassmann origin). The (formal) prime spectrum, $\operatorname{Spec} \Lambda$, is just a one-point superspace with the algebra of sections of the structure sheaf isomorphic to $\Lambda$. Let $X$ be a $\Lambda$-superspace. The fact that the algebras of sections of the structure sheaf on $X$ are all algebras over $\Lambda$ means the existence of a canonical fixed morphism of locally ringed superspaces $X \rightarrow \operatorname{Spec} \Lambda$. On the other hand, any morphism of the form $X \rightarrow \operatorname{Spec} \Lambda$, where $X$ is a geometric superspace, gives rise to a structure of a $\Lambda$-superspace on $X$. Therefore, it may happen that the entire theory of $\Lambda$-supermanifolds is not a "generalisation" of the theory of BLK-supermanifolds, but rather a special case of the theory of BLK superfibre bundles (with base infinite-dimensional in the odd sector, in general).

This idea was advertised by Leites, mostly in private commmunications. Some initial work has been done by Penkov [99].

\section{SUPERGROUPS AND LIE SUPERALGEBRAS}

A supergroup is a group object in a category of supermanifolds. In other words, a supergroup is a quadruple $(G, \mu, \iota, \varepsilon)$, where $G$ is a (smooth or analytical) supermanifold, $\mu: G \times G \rightarrow G, \iota: G \rightarrow G$, and $\varepsilon: * \rightarrow G$ are supermanifold morphisms satisfying the natural properties, the symbol $\times$ stands for the direct product in a category of supermanifolds (known to exist), and $*$ is a terminal object in the same category (a supermanifold of dimension $(0,0))[31,34]$.

A group object in the category of DeWitt supermanifolds is called a DeWitt supergroup, otherwise it is called a Rogers supergroup. Of course, this distinction makes no sense in the category of BLK supermanifolds. Supergroups in the BLK category are also referred to as graded Lie groups.

Of course, one should be cautious enough to remember that since morphisms between supermanifolds are not completely specified by the underlying set-theoretic map- 
pings (apart from the "naive" approach), the structure of a supergroup is not determined solely by the underlying group structure on the manifold of points. However, it is determined by a family of (Lie) group structures on the manifolds of $q$-points. (An extensive discussion of Lie groups of points is contained in the DeWitt's book [54]. He called those Lie groups $q$-skeletons of the supergroup. Another presentation of the subject is to be found in [31].)

Lie superalgebras are just graded versions of Lie algebras. A free $\Lambda$-module is a Lie superalgebra if it is endowed with a bi- $\Lambda$-linear super Lie bracket, which is graded anticommutative and satisfies the graded Jacobi identity.

To every supergroup $G$ there is associated a Lie superalgebra, consisting of all left-invariant graded vector fields on $G$ endowed with the graded version of the familiar Lie bracket (supercommutator of vector fields). A vector field $X$ on $G$ (that is, a graded derivation of the structure sheaf) is left-invariant if ( $\operatorname{Id} \otimes X) \mu^{\sharp} f=\mu^{\sharp}(X f)$ for each superfunction $f$, where $\mu$ stands for the multiplication morphism and the tensor product of algebras of sections must be understood as the completed topological tensor product (in a different sense for different sorts of supermanifolds - smooth, analytic et cetera).

The body, $\mathfrak{g}_{B}$, of a Lie superalgebra over an augmented graded-commutative algebra $\Lambda$, is a Lie superalgebra over the basic field $\mathbb{K}$. Therefore, a Lie superalgebra $\mathfrak{g}$ over an augmented graded-commutative algebra $\Lambda$ (in particular, any algebra of Grassmann origin, say, a Grassmann algebra) is, from the viewpoint of deformation theory, a deformation of its body over $\operatorname{Spec} \Lambda$, see [57].

A super version of the Lie-Cartan theorem for BLK supermanifolds was obtained by Berezin and Leites [27] and Kostant [79]: every finite-dimensional Lie $\mathbb{K}$-superalgebra is isomorphic to the Lie superalgebra of an essentially unique supergroup $G$ such that the underlying Lie group of $G$ is simply connected. (The first ever approximation to this result, at the level of formal supergroups, was obtained in [26].) Later this result was extended by a number of authors to supergroups over "algebras of supernumbers" $\Lambda$ belonging to various classes $[122,39,17,141,100,102,111]$. The most general result of this kind was obtained by the author $[102,111]$ : every finite-dimensional Lie $\Lambda$-superalgebra, where $\Lambda$ is a complete locally multiplicatively convex graded commutative local algebra, is isomorphic to the Lie superalgebra of an essentially unique $\Lambda$ supergroup $G$ such that the underlying Lie group of $G$ is simply connected. Moreover, $G$ is a DeWitt supergroup. (A topological algebra $A$ is called locally multiplicatively convex, or locally m-convex, or just l.m.c., if it is isomorphic to a subalgebra of the direct product (with the Tychonoff topology) of a family of normed algebras. Equivalently: the topology of $\boldsymbol{A}$ is determined by the family of all continuous seminorms $p$ with the property $p(x y) \leqslant p(x) p(y)$. (Such seminorims are called submultiplicative 
ones.) Still another equivalent definition: convex circled multiplicative subsemigroups of $A$ form a neighbourhood basis at zero. The theory of such algebras was developed in [3] and [93]; see also [61].) In other words, every deformation of a finite-dimensional Lie superalgebra over the prime spectrum of a complete l.m.c. graded commutative local algebra (GLAM algebra - from Graded Local Arens-Michael) is enlargable to a DeWitt supergroup. Heuristically, deformations of finite-dimensional graded Lie algebras come from deformations of corresponding graded Lie groups. We hope that DeWitt supergroups can be thought of as deformations of graded Lie groups over spectra of graded-commutative algebras. Thus, DeWitt theory might fit in the bed of deformation theory of graded Lie groups. To our knowledge, there is no mathematically rigorous approach yet.

Here is an example due to DeWitt $[\mathbf{5 4}, \mathbf{5 5}]$ of a Lie supergroup in dimension $(0,1)$ which does not come from any graded Lie group. Define a multiplication morphism, inversion morphism, and the unity morphism on the supermanifold $\Lambda^{(0,1)}$ as

$$
\theta * \eta=\theta+\eta+c \theta \eta ; \quad \theta^{-1}=-\theta ; \quad e=0
$$

( $c$ being an odd constant.) The Lie superalgebra of the above supergroup it is isomorphic as a graded $\Lambda$-module to $\Lambda \otimes \mathbb{K}^{0,1} \simeq \Lambda^{1} X+\Lambda^{1} X$, where $X$ is the odd generator, with the only (anti)commutation relation $[X, X]=2 c X$. This is a deformation, over $\operatorname{Spec} \Lambda$, of the Abelian Lie superalgebra of dimension $(0,1)$. (Just set $c=0$.)

Surprisingly, the above results are no longer true in infinite dimensions. A recent result of the author [110] indicates that Rogers supergroups may be indispensable in some situations. An example has been constructed of an infinite-dimensional Lie superalgebra $\mathfrak{g}$ over the DeWitt supernumber algebra $\Lambda_{\infty}$ such that $\mathfrak{g}$ comes from a Rogers supergroup and comes from no DeWitt supergroup. Another example shows that an infinite-dimensional Lie $\Lambda_{\infty}$-superalgebra can be (even Rogers) non-enlargable, while the body is an enlargable Banach-Lie algebra.

For some other aspects of super Lie theory, consult $[58,98,113,117,118]$.

\section{LINKS BETWEEN SUPERMATHEMATICS AND "ClASSICAL" MATHEMATICS}

From the very beginning, superanalysis and supergeometry were shaped after classical analysis and geometry. In some cases the process of "superization" constitutes no difficulties and is a mere formal rewording of a definition or a proof. In other cases (such as superdeterminant) a good portion of inventiveness was needed, and "superization" proved to be highly nontrivial. Anyway, the ease with which one can "superize" numerous classical concepts and results, suggests that the process of "superization" can be given a metamathematical form; however, very little has been done in this direction. (We shall discuss the possibility of such a kind in our concluding remarks.) 
Of course, many objects of superanalysis can be themselves interpreted as "classical", "non-super" objects. One striking example of this kind is Batchelor's theorem $[20,32]$, which puts BLK supermanifolds and exterior algebras on vector bundles in a one-to-one correspondence. Even before Batchelor's result, it was known among the physicists that the structure sheaf of a superspace (that is, the totality of superfunctions, or superfields) can be thought of as the sheaf of differential forms on a smooth manifold, and, say, the famous paper by Witten [146] was written in this context. Another surprising result by Batchelor [21] enables one to view BLK supermanifolds as pairs of ordinary manifolds.

Atiyah suggested [5] that "super" analysis and geometry may be the limit of ordinary analysis and geometry on a smooth manifold; to my knowledge, the idea has not been developed by anyone.

Now I wish to proceed to the discussion of another idea, belonging to Manin [87]. He points to many occasions in theoretical physics where bosonic effects arose in the presence of infinitely many fermionic sources. Mathematically, it could mean that fermionic coordinates are primary with respect to the bosonic ones; in particular, Manin suggested that

the even geometry $=$ a collective effect in $\infty$-dimensional odd geometry

and remarked that this idea "is still awaiting the precise mathematical theory". He writes: "Our usual four space-time coordinates possibly reflect only the collective effect, a coherent state of the world of quantum fields at low temperature, which makes possible the existence of life in its usual form."

I wish to put forward now the rudiments of a possible mathematical explanation of such a phenomenon. We need a technique well suited for handling limiting processes, and it comes as no surprise that at this stage another brand of non-classical analysis comes into being - Nonstandard Analysis.

All constructions and results can be reshaped by means of ultraproducts. However, it is our belief that a nonstandard approach is more beneficial.

\section{NonstandaRd Hulls}

Nonstandard Analysis is an invention of Abraham Robinson [119], and it is of about the same age as the subject of my talk. The main idea of Nonstandard Analysis is to proceed from classical objects - such as groups $G$, algebras $A$, topological spaces $X$, the real line $\mathbb{R}$ and so on - to their nonstandard enlargments, denoted by putting a star at the upper left corner: ${ }^{\star} G,{ }^{\star} A,{ }^{\star} X,{ }^{\star} \mathbb{R}$. Those enlargments are models of the above structures with respect to an appropriate language, a so-called Higher Order Language, with variables running over relations of any order on the original 
set. The main tool of nonstandard analysis is the so-called Transfer Principle, which implies that a statement $P$ about a structure $A$, expressed in terms of the Higher Order Language, is true if and only if a statement ${ }^{\star} P$ about the enlargement ${ }^{\star} \mathfrak{M}$, obtained by putting stars on all the constants of $P$, is true. Every object of the form ${ }^{\star} A$ contains $A$ (in an appropriate sense), and on nontrivial occasions (namely, if $A$ is infinite) one has $A \subsetneq{ }^{*} A$. For example, the nonstandard real line ${ }^{\star} \mathbb{R}$ forms, from a classical viewpoint, a non-Archimedean ordered field; in particular, it contains infinitely small and infinitely large elements. At the same time, in a model-theoretic sense, ${ }^{\star} \mathbb{R}$ shares all the properties of the ordinary real line, $\mathbb{R}$. Objects of the form ${ }^{\star} A$ are called standard, and objects which are themselves elements of standard objects are called internal. All other objects are called external. For example, the object $\star \mathbb{R}$ is standard; an infintesimal non-zero element $\alpha \in{ }^{\star} \mathbb{R}$ is internal but nonstandard; and the totality of all infinitesimal elements, $\mu_{\mathbb{R}}(0)=\left\{x \in{ }^{\star} \mathbb{R}: \forall n \in \mathbb{N} \quad|x|<1 / n\right\}$ is an external set. (The Transfer Principle applies to internal objects only, so all knowledge about external sets is obtained in a roundabout way.) The most important - and even in a certain sense unique - way of constructing nonstandard enlargments is via ultrapowers, so the whole theory can be rewritten in the language of ultrapowers; however, it is usually much more difficult to prove things about ultrapowers.

Let us discuss the concept of the nonstandard hull [139]. Let $E \in{ }^{\star} \mathfrak{M}$ be in internal normed space. By fin $E$ one denotes the (external) set of all the norm finite elements of $E$, that is,

$$
\text { fin } E=\{x \in E:\|x\| \text { is finite }\} \text {. }
$$

Similarly, one puts

$$
\mu_{E}(0)=\{x \in E:\|x\| \approx 0\} .
$$

Both fin $E$ and $\mu_{E}(0)$ are linear subspaces of $E$ over $\mathbb{R}$. The quotient linear space $\widehat{E}:=$ fin $E / \mu_{E}(0)$ is endowed with a norm as follows. Let $\pi_{E}$ : fin $E \rightarrow \widehat{E}$ stand for the quotient homomorphism. Then the following rule is easily verified to define $\mathrm{E}$ on $\widehat{E}$ a norm in a correct way:

$$
\left\|\pi_{E} x\right\|_{\widehat{E}}=\text { st }\|x\|_{E}, x \in \text { fin } E
$$

where st $:$ fin $\mathbb{R} \rightarrow \mathbb{R}$ is the standard part map. The standard normed space $\widehat{E}$ is Banach. It is called the nonstandard hull of $E$.

Now let $A \in \star^{\star} \mathfrak{M}$ be a normed algebra endowed with a submultiplicative norm. In this case, fin $A$ is an $\mathbb{R}$-algebra and $\mu_{E}(0)$ is a two-sided ideal in it; thus, the hull $\widehat{A}$ is a standard $\mathbb{R}$-algebra. The norm on $\widehat{A}$ is submultiplicative and complete. The algebra $\hat{A}$ is "of the same type" as $A$; to be more precise, if $A$ belongs to some variety of algebras, then so does $\widehat{A}$. In particular, all the (standard) identities which hold in $A$ are valid in $\hat{A}$ as well, and if $A$ is an associative (unital, Lie, ...) algebra then so is $\widehat{A}$. 
The construction of the nonstandard hull will be applied by us only in the case where the space $E$ (or algebra $A$ ) is standard. In this situation, it makes sense also for locally convex spaces and algebras [139].

\section{COLLECTIVE EVEN EFFECTS IN INFINITE-DIMENSIONAL ODD SUPERSPACES: SPATIAL RESULTS}

Consider an infinite-dimensional Grassmann algebra $\Lambda(\infty)$ - or, equivalently, a purely odd supermanifold of dimension $(0, \infty)$. Put an $l_{1}$-type norm on the algebra. It is a local algebra, its prime spectrum is one-pointed; indeed, if $\theta$ is a quasi-nilpotent element, then for every character $\chi$ one has $\chi(\theta)=0$. Recall that within the paradigm of functional analysis, characters of algebras of functions correspond to points, so the even geometry associated to a supermanifold of dimension $(0, \infty)$ is trivial.

Now suppose that every thing happens within a nonstandard model of analysis, ${ }^{*} \mathfrak{M}$. Fix an infinitely large positive integer $\kappa$. For any $i \in[1, \kappa] \subset \star \mathbb{N}$, let $\theta_{i}:=\xi_{2 i-1} \xi_{2 i}$. Each $\theta_{i}$ is an even 2-nilpotent element with unit norm. Put

$$
\theta=(\operatorname{Card} I)^{-1} \sum_{i \in I} \theta_{i}
$$

The key statement is that for any $n \in \mathbb{N}$ standard, $\left\|\theta^{n}\right\| \approx 1$.

Indeed, $\left\|\theta^{n}\right\| \leqslant\|\theta\|^{n}=1$. On the other hand, of the totality of $\eta^{n}$, where $\eta:=\operatorname{Card} I$, monomials in the representation of $\theta^{n}$, at most $C_{n}^{2} \eta^{n-1}$ monomials are vanishing. Since it is easy to see that the contribution to the norm of the sum from the rest of monomials is the same for any two of them,

$$
1 \geqslant\left\|\theta^{n}\right\| \geqslant \eta^{-n}\left[\eta^{n}-C_{n}^{2} \eta^{n-1}\right]=1-C_{n}^{2} / \eta \approx 1
$$

Therefore, there are sufficiently many even elements of spectral radius 1 in the nonstandard hull algebra $\widehat{\Lambda(\nu)}$; but it means that there are sufficiently many characters on the same algebra. Denote the space of all characters of $\widehat{\Lambda(\nu)}$ by $X_{0}$. One can show that $X_{0}$ is an infinite-dimensional unseparable compact space. This space contains a topological copy of the cube $I^{n}$ for each $n$.

One can construct a superspace $X$ starting with the graded-commutative Banach algebra $\widehat{\Lambda(\nu)}$. The construction is a version of the construction of the prime spectrum of a graded ring, while the Zariski topology is replaced by the stronger Gel'fand (weak) topology, and necessary completions of all "superfunction algebras" are made. In other terms, it is a solution to a certain universal problem. The resulting superspace, $X$, is "very big," and it is infinite-dimensional in the odd sector as well. 
In an eye-catching form, this result looks as follows:

$$
\lim _{q \rightarrow \infty}(0, q)=(\infty, \infty)
$$

One can construct the even sector of the superspace $X$ as the geometric space $X_{\text {red }}$, the quotient of $X$ by the sheaf of all quasinilpotents. The space $X_{\text {red }}$ is called the reduced subsuperspace of $X$ (see [88]).

The above construction was suggested by the author $[104,105]$. With quite obvious modifications, it extends to the case of an arbitrary locally convex gradedcommutative algebra $\Lambda$, and it is functorial (provided that all nonstandard enlargements are ultrapowers with respect to a fixed ultrafilter). One of the functors arising is a contravariant functor from the category $\mathcal{G}$ of locally convex graded-commutative algebras and continuous even homomorphisms to the category $\mathcal{T} y$ ch of Tychonoff topological spaces and continuous mappings. The category $\mathcal{G}^{\text {op }}$ opposite to $\mathcal{G}$ can be thought of as a category of superspaces of dimension $(0, \infty)$, and the resulting functor from $\mathcal{G}^{\text {op }}$ to $\mathcal{T}$ ych is covariant.

Two other functors arising send $\mathcal{G}^{o p}$ to the category of geometric (super)spaces.

In [109] it was shown that the geometric space $X$ image of the algebra $\Lambda=\Lambda l_{1}$ (the exterior algebra on countably many odd generators, endowed with the strongest locally convex topology making the set of generators bounded) under the nonstandard hull functor carries a certain analytic structure. Namely, there exists a homeomorphic embedding $\phi$ of the closed unit disk $D \subseteq \mathbb{C}$ into $X$ in such a way that for every $a \in \widehat{\bigwedge l}$ the composition of $\phi$ and the Gelfand transform $\widehat{a}$ of $a$ is a holomorphic map from $D$ to $\mathbb{C}$.

\section{Graded Lie-Cartan pairs}

Here we shall briefly consider the concept of a (graded) Lie-Cartan pair $[74,75,66$, 67] which is one "non-spatial" way to deal with noncommutative differential geometry [48]. A Lie-Cartan pair $(L, A)$ consists of a graded-commutative algebra $A$, a Lie (super)algebra $L$ (not in general a Lie superalgebra over $A$ in our sense) which is a graded left unital $A$-module (not necessarily free over $A$ ), and a fixed (even) Lie homomorphism $L \rightarrow \operatorname{Der} A$, subject to the following axioms.

(i) $a(\xi b)=(a \xi) b$ for all $a, b \in A$ and $\xi \in L$;

(ii) $[\xi, a \eta]=(-1)^{\widetilde{\xi a}} a[\xi, \eta]+(\xi a) \eta$ for all $\xi, \eta \in L$ and $a \in A$.

(Here [,] stands for the supercommutator in $L$, and the usual conventions about homogenuous elements are assumed.) (The basic case to bear in mind is that where $A=C^{\infty}(X)$ is the algebra of smooth functions on a finite-dimensional manifold $X$, and $L=\operatorname{vect}(X)$ is the Lie algebra of smooth vector fields on $X$.) 
Given a graded unital $A$-module $E$, one can introduce in a formal way the concept of an $E$-connection as a certain linear map $L \rightarrow$ End $E$, then to define the curvature, and number of classical operators (such as the exterior derivative), thus enabling one to develop a version of differential geometry in an algebraic (non-spatial) framework. An advantage of such an approach is that it embraces both classical differential geometry on smooth manifolds and supergeometry. In particular, the case where $A$ is a Grassmann algebra and $L$ is the algebra of all graded derivations of $A$ corresponds to the purely odd (fermionic) differential geometry.

Of course, given a (graded) commutative aglebra $A$, the pair $(\operatorname{Der} A, A)$ forms a Lie-Cartan pair. One of the difficulties in such a general case is that it is not clear whether $A$ admits nontrivial derivations at all.

The technique of nonstandard hulls (or, equivalently, ultraproducts) permits one, firstly, to construct new examples of nontrivial Lie-Cartan pairs, and secondly, to push on further the program of "bosonisation" of purely odd geometry.

Consider a Lie-Cartan pair $(L, A)$ and assume that the algebra $A$ of "functions" carries a locally convex topology. (This is invariably the case in all "classical" examples. Whether or not the topology on algebras of superfunctions in the purely odd case comes from some natural causes, and what the real significance of such a topology is, we know not. We suggest that the presence of a topology on a function algebra is a way to encode its "spatiality.") We shall assume that the fixed homomorphism $L \rightarrow \operatorname{Der} A$ is a monomorphism. (The cases where the kernel of the above homomorphism is non-zero, play a certain rôle in theory [47], and even the degenerate case where this kernel is $L$ does so - the so-called depletions of Lie-Cartan pairs. However, we wish to exclude such cases from consideration.)

Denote by fin $L$ the set of all $\xi \in L$ such that $\xi($ fin $A) \subseteq$ fin $A$, and by $\mu_{L}(0)$ the set of all $\xi \in L$ with $\xi x \in \mu_{A}(0)$ for all $x \in$ fin $A$. One can check that fin $L$ is a Lie subalgebra of $L$, and $\mu_{L}(0)$ is a Lie ideal in fin $L$. Denote by $\widehat{L}$ the quotient Lie algebra fin $L / \mu_{L}(0)$. The monomorphism fin $L \rightarrow \operatorname{Der}$ fin $A$ factors through $\mu_{L}(0)$, giving rise to a monomorphism $\widehat{L} \rightarrow \operatorname{Der} \widehat{A}$. It is easy to check the axioms of a Lie-Cartan pair for the pair $(\widehat{L}, \widehat{A})$. We call it the nonstandard hull of the pair $(L, A)$.

One can show now, using the known description of the Lie superalgebra of derivations of a Grassmann algebra [73] that the nonstandard hull of the Lie-Cartan pair $\left(\operatorname{Der} \wedge l_{1}, \wedge l_{1}\right)$ of infinite-dimensional fermionic differential geometry is highly nontrivial. In particular, the nonstandard hull algebra of derivations $\widehat{\operatorname{Der} \Lambda} l_{1}$ is rich in the following sense: if an element $a \in \widehat{\Lambda l_{1}}$ is such that for every $\xi \in \widehat{D e r} \Lambda l_{1}$ one has $\xi a=0$, then $a \in \mathbb{K}$ is a constant. This result gives hope that the even differential geometry of the nonstandard hull $\widehat{\Lambda l_{1}}$ is substantial (both in present "nonspatial" version, and in "spatial" one, reshaped for the geometric space $X_{\text {red }}$ ). See [112] for more 
details.

\section{SOME REMARKS}

It is our belief that further development of analysis on superspaces will be guided by two fundamental ideas: "superization" (giving the process of "superization" of even concepts a metamathematical form), and "bosonization" (Manin's idea discussed above). It is plausible that the basic notions of superanalysis in the long run will be put on a topos-theoretic ground [71]. (An impressive program is sketched by Molotkov in [95].) One of the basic notions in this connection may be that of a "pointless space" (locale, frame) - or, rather, an appropriate super analogue of it. Curiously enough, Berezin himself insisted that the "true" notion of a supermanifold should be "pointless", and he objected to publishing his joint results with Leites on supermanifolds, considering these as a preliminary version. (See [83].)

If superanalysis and supergeometry are to become a kind of nonstandard model theory, then "superization" of some ordinary notion will look very much like the procedure of "putting a star" in nonstandard analysis [119] or the "up" procedure in Boolean-valued analysis $[140,81]$.

A closely related powerful development is braided category theory, offering a new look at quantum groups $[\mathbf{7 2}, \mathbf{8 6}, \mathbf{8 9}]$. Supermathematics finds a place inside this theory as a "testing ground" for new concepts; it is a case both not quite trivial and yet tame, where "everything works well."

Synthetic geometry [94] may turn out to be another very important tool in establishing a precise relationship between purely odd superspaces and spaces of usual analysis. The mathematical reason for this is very simple: the Weil algebra $W(n)$, playing a major rôle in synthetic analysis and geometry (an associative unital algebra freely generated by $n$ 2-nilpotent elements), plainly embeds into the even part of the Grassmann algebra $\Lambda(2 n)$. See the interesting paper [147], establishing the first notions of emerging synthetic supergeometry. It may turn out that the nonstandard hull technique is also applicable to Weil algebras, thereby leading to an interplay between the two kinds of infinitesimals.

An account of unsolved problems in supermanifold theory is contained in the paper by Leĭtes [84].

\section{REFERENCES}

[1] A.L. Almorox, 'Supergauge theories in graded manifolds', in Differential geometric methods in mathematical physics, Lecture Notes Mathematics 1251 (Springer-Verlag, Berlin, Heidelberg, New York, 1987), pp. 114-136. 
[2] A. Arai, 'Some remarks on scattering theory in supersymmetric quantum mechanics', $J$. Math. Phys. 28 (1987), 472-476.

[3] R. Arens, 'A generalization of normed rings', Pacif. J. Math. 2 (1952), 455-471.

[4] R. Arnowitt, P. Nath and B. Zumino, 'Superfield densities and action principle in superspace', Phys. Lett. B 56 (1975), 81-84.

[5] M. Atiyah, 'A commentary on the article of Manin', Lecture Notes in Math. 1111 (1985), p.103.

[6] A.M. Baranov, Yu.I. Manin, I.V. Frolov and A.S. Schwarz, 'A superanalog of the Selberg trace formula and multiloop contributions for fermionic strings', Comm. Math. Phys. 111 (1987), 373-392:

[7] C. Bartocci, Elementi di geometria globale delle supervarietà, (in Italian), Ph.D. Thesis (University of Genova, 1991).

[8] C. Bartocci and U. Bruzzo, 'Some remarks on the differential-geometric approach to supermanifolds', J. Geom. Phys. 4 (1987), 391-404.

[9] C. Bartocci and U. Bruzzo, 'Cohomology of supermanifolds', J. Math. Phys. 28 (1987), 2363-2368.

[10] C. Bartocci and U. Bruzzo, 'Cohomology of the structure sheaf of real and complex supermanifolds', J. Math. Phys. 29 (1988), 1789-1795.

[11] C. Bartocci and U. Bruzzo, 'Existence of connections on superbundles', Lett. Math. Phys. 17 (1989), 61-68.

[12] C. Bartocci and U. Bruzzo, 'Super line bundles', Lett. Math. Phys. 17 (1989), 263-274.

[13] C. Bartocci, U. Bruzzo and D. Hernández Ruipérez, 'A remark on a new category of supermanifolds', J. Geom. Phys. 6 (1989), 509-516.

[14] C. Bartocci, U. Bruzzo and D. Hernández Ruipérez, The geometry of supermanifolds (Kluwer Acad. Publ., Dordrecht, 1991).

[15] C. Bartocci, U. Bruzzo, D. Hernández Ruipérez and V.G. Pestov, 'On an axiomatic approach to supermanifolds', (in Russian), Dokl. Akad. Nauk SSSR 321 (1991), 649-652.

[16] C. Bartocci, U. Bruzzo, D. Hernández Ruipérez and V.G. Pestov, 'Foundations of supermanifold theory: the axiomatic approach', Differential Geom. Appl. 3 (1993), 135-155.

[17] C. Bartocci, U. Bruzzo and G. Landi, 'Chern-Simons forms on principal superfibre bundles', ISAS preprint 109/87/FM, Trieste, 1987.

[18] M. Batchelor, 'Two approaches to supermanifolds', Trans. Amer. Math. Soc. 258 (1980), 257-270.

[19] M. Batchelor, 'Graded manifolds and supermanifolds', in Mathematical Aspects of Superspace, (H.-J. Seifert et al, Editors) (D. Reidel Publ. Co., 1984), pp. 91-133.

[20] M. Batchelor, 'Graded manifolds and vector bundles: a functorial correspondence', $J$. Math. Phys. 26 (1985), 1578-82.

[21] M. Batchelor, 'Graded manifolds and pairs', in Differential geometric methods in mathematical physics, Lecture Notes in Mathematics 1251 (Springer-Verlag, Berlin, Heidelberg, New York, 1987), pp. 65-72.

[22] F.A. Berezin, Method of Second Quantization (Academic Press, New York, 1966). 
[23] F.A. Berezin, 'Automorphisms of a Grassmann algebra', Math. Notes 1 (1967), 180-184.

[24] F.A. Berezin, Introduction to algebra and analysis with anticommuting variables, (in Russian) (Moscow State University, Moscow, 1983).

[25] F.A. Berezin, Introduction to superanalysis, (A.A. Kirillov, Editor) (D. Reidel Publ. Co, Dordrecht-Boston, MA, 1987).

[26] F.A. Berezin and G.I. Kats, 'Lie groups with commuting and anticommuting parameters', Math. USSR Sb. 11 (1970), 311-326.

[27] F.A. Berezin and D.A. Leites, 'Supermanifolds', Soviet Math. Dokl. 16 (1975), 1218-1222.

[28] F.A. Berezin and M.S. Marinov, 'Particle spin dynamics as the Grassmann variant of classical mechanics', Ann. Physics 104 (1977), 336-362.

[29] J. Bernstein and D. Leĭtes, 'Integral forms and Stokes' formula on supermanifolds', Funct. Anal. Appl. 11 (1977).

[30] J. Bernstein and D. Leǐtes, 'How to integrate differential forms on supermanifolds', Funct. Anal. Appl. 11 (1977), 219-221.

[31] J. Bernstein and D. Leĭtes, 'Lie superalgebras and supergroups', Rep. Dept. Math. Univ. Stockholm 14 (1988), 132-260.

[32] R.J. Blattner and J.H. Rawnsley, 'Remarks on Batchelor's theorem', in Mathematical Aspects of Superspace (D. Reidel Publ. Co., 1984), pp. 161-171.

[33] C.P. Boyer and O.A. Sánchez Valenzuela, 'Some problems of elementary calculus in superdomains (with a survey of the theory of supermanifolds)', Aportaciones Mat. 5 (1988), 111-143.

[34] C.P. Boyer and O.A. Sánchez Valenzuela, 'Lie supergroup actions on supermanifolds', Trans. Amer. Math. Soc. 323 (1991), 151-173.

[35] C. Boyer and S. Gitler, 'The theory of $G^{\infty}$-supermanifolds', Trans. Amer. Math. Soc. 285 (1984), 241-267.

[36] U. Bruzzo, 'Geometry of rigid supersymmetry', Hadronic J. 9 (1986), 25-30.

[37] U. Bruzzo, 'Supermanifolds, supermanifold cohomology, and super vector bundles', in Differential geometric methods in theoretical physics, NATO ASI Ser. 250 (Kluwer, Dordrecht, 1988), pp. 417-440.

[38] U. Bruzzo, 'Berezin integration on DeWitt supermanifolds', Israel J. Math. 80 (1992), 161-169.

[39] U. Bruzzo and R. Cianci, 'An existence result for super Lie groups', Lett. Math. Phys. 8 (1984), 279-88.

[40] U. Bruzzo and R. Cianci, 'On the structure of superfields in a field theory on a supermanifold', Lett. Math. Phys. 11 (1986), 21-26.

[41] U. Bruzzo and D. Hérnandez Ruipérez, 'Characteristic classes of super vector bundles', J. Math. Phys. 30 (1989), 1233-1237.

[42] P. Bryant, 'DeWitt supermanifolds and infinite-dimensional ground rings', J. London Math. Soc. 39 (1989), 347-368.

[43] J.L. Carr, 'Spin structures and superworldsheet topology', Classical Quantum Gravity 6 (1989), 125-140. 
[44] Y. Choquet-Bruhat, 'Mathematics for classical supergravities', in Lecture Notes in Math. 1251 (Springer-Verlag, New York, 1987), pp. 73-90.

[45] Y. Choquet-Bruhat, Graded bundles and supermanifolds, Monographs and Textbooks in Physical Sciences (Bibliopolis, Naples, 1990).

[46] Y. Choquet-Bruhat and C. DeWitt-Morette, Analysis, manifolds and physics. Part II: 92 Applications (North-Holland, Amsterdam, Oxford, New York, Tokyo, 1989).

[47] R. Coqueraux, A. Jadczyk, and D. Kastler, 'Differential and integral geometry of Grassmann algebras', Rev. Math. Phys. 3 (1991), 63-99.

[48] A. Connes, 'Non-commutative differential geometry', Publ. Math. IHES 62 (1985), 41-144.

[49] A. Connes, 'On the Chern character in $\theta$ summable Fredholm modules', Comm. Math. Phys. 139 (1991), 171-181.

[50] L. Corwin, Y. Ne'eman, and S. Sternberg, 'Graded Lie algebras in mathematics and physics (Bose-Fermi symmetry)', Rev. Modern Phys. 47 (1975), 573-603.

[51] J. Dell and L. Smolin, 'Graded manifold theory as the geometry of supersymmetry', Commun. Math. Phys. 66 (1979), 197-221.

[52] M. Demazure and P. Gabriel, Introduction to algebraic geometry and algebraic groups (North-Holland, Amsterdam, New York, Oxford, 1980).

[53] B.S. DeWitt, Dynamical theory of groups and fields (Gordon and Breach, New York, London, Paris, 1965).

[54] B.S. DeWitt, Supermanifolds (Cambridge University Press, London, 1984).

[55] B.S. DeWitt, 'The spacetime approach to quantum field theory', in Relativité, groupes et topologie II. Les Houches, Session XL (1983), (B.S. DeWitt and R. Stora, Editors) (North-Holland, Amsterdam, Oxford, New York, Tokyo, 1986), pp. 383-738.

[56] A.A. Faustov, On the Banach-Grassmann algebras (Institute of Mathematics Preprint, Novosibirsk, 1991).

[57] B.L. Feigin and D.B. Fuks, 'Lie groups and algebras', (in Russian), in Contemporary problems of mathematics. Fundamental directions 21 (VINITI, Moscow, 1988), pp. 121-213.

[58] H. Fetter and F. Ongay, 'An explicit classification of $(1,1)$-dimensional Lie supergroup structures', in Proc. XIX Int. Conf. on Group Theor. Methods in Physics (Salamanca, 1992). Anales de Física de la Real Soc. Esp. de Fis. Vol. II, 1993, pp. 261-269.

[59] S.J. Gates, M.T. Grusaru, M. Roček and W. Siegel, Superspace, or One Thousand and One Lessons in Supersymmetry (Benjamin/Cummings, Reading (Mass.), 1983).

[60] P. Green, 'On holomorphic graded manifolds', Trans. Amer. Math. Soc. 85 (1982), 587-590.

[61] A.Ya. Helemskir, Banach and polynormed algebras. General theory, representations, homology, (in Russian) (Nauka, Moscow, 1989).

[62] D. Hernández Ruipérez and J. Muñoz Masqué, 'Global variational calculus on graded manifolds, I: graded jet bundles, structure 1-form and graded infinitesimal contact transformations', J. Math. Pures Appl. 63 (1984), 283-309.

[63] D. Hernández Ruipérez and J. Muñoz Masqué, 'Construction intrinsèque du faisceau de Berezin d'una variété graduée', C.R. Acad. Sci. Paris Sér. I 301 (1985), 915-918. 
[64] J. Hoyos, M. Quirós, J. Ramírez Mittelbrunn and F.J. de Urríes, 'Generalized supermanifolds. I-III', J. Math. Phys. 25 (1984), 833-854.

[65] V.D. Ivashchuk, 'On annihilators in infinite-dimensional Grassmann-Banach algebras', (in Russian), Teoret. Mat. Fiz. 79 (1989), 30-40.

[66] A. Jadczyk and D. Kastler, 'Graded Lie-Cartan pairs I', Rep. Math. Phys. 25 (1987), $1-51$.

[67] A. Jadczyk and D. Kastler, 'Graded Lie-Cartan pairs II. The fermionic differential calculus', Ann. Physics 179 (1987), 169-200.

[68] A. Jadezyk and K. Pilch, 'Classical limit of CAR and self-duality in the infinite-dimensional Grassmann algebra', in Quantum theory of particles and fields, (B. Jancewicz and J. Lukierski, Editor) (World Scientific, Singapore, 1983).

[69] A. Jadczyk and K. Pilch, 'Superspaces and supersymmetries', Commun. Math. Phys. 78 (1981), 373-390.

[70] A. Jaffe and F. Quinn, "Theoretical mathematics": toward a cultural synthesis of mathematics and theoretical physics', Bull. Amer. Math. Soc. 29 (1993), 1-13.

[71] P.T. Johnstone, Topos theory (Academic Press, London, New York, San Fransisco, 1977).

[72] A. Joyal and R. Street, Braided tensor categories (Macquarie University preprint).

[73] V.G. Kac, 'Lie superalgebras', Adv. in Math. 26 (1977), 8-96.

[74] D. Kastler, 'Introduction to Alain Connes' non-commutative differential geometry', in Fields and Geometry 1986. Proc. XXIInd Winter School and Workshop on Theor. Phys. Karpacz, Poland., (A. Jadczyk, Editor) (World Scientific), pp. 387-465.

[75] D. Kastler and R. Stora, 'Lie-Cartan pairs', J. Geom. Phys. 2 (1985), 1-31.

[76] A.Yu. Khrennikov, 'Functional superanalysis', Russian Math. Surveys 43 (1988), 103-137.

[77] S. Klimek and A. Lesniewski, 'Pfaffians on Banach spaces', J. Funct. Anal. 102 (1991), 314-330.

[78] Y. Kobayashi and Sh. Nagamachi, 'Usage of infinite-dimensional nuclear algebras in superanalysis', Lett. Math. Phys. 14 (1987), 15-23.

[79] B. Kostant, 'Graded manifolds, graded Lie theory, and prequantization', in Differential geometric methods in mathematical physics, Lecture Notes Mathematics 570 (Springer-Verlag, Berlin, Heidelberg, New York, 1977), pp. 177-306.

[80] J. Kupsch, 'Measures for fermionic integration', Fortschr. Phys. 35 (1987), 415-436.

[81] A.G. Kusraev and S.S. Kutateladze, Nonstandard methods of analysis, (in Russian) (Nauka, Novosibirsk, 1990).

[82] D.A. Leĭtes, 'Introduction to the theory of supermanifolds', Russian Math. Surveys 35 (1980), 1-74.

[83] D.A. Leĭtes, The theory of supermanifolds, (in Russian) (Karelian Branch Acad. Sciences U.S.S.R., Petrozavodsk, 1983).

[84] D.A. Leǐtes, 'Some open problems in theory of supermanifolds', Duke Math. J. 54 (1987), 649-656.

[85] D. Leĭtes, Yu. Kochetkov and A. Weintrob, 'New invariant differential operetors on supermanifolds and pseudo-(co)homology', in General topology and its applications Proc. 
5th Northeast Conf., NY, (1989)., Lect. Notes Pure and Appl. Math. 134, (1991), pp. 217-238.

[86] S. Majid, Braided groups (DAMTP preprint 90-42, 1990).

[87] Y. Manin, 'New dimensions in geometry', in Lecture Notes in Math. 1111 (Springer-Verlag, New York, 1985), pp. 59-101.

[88] Yu.I. Manin, Gauge field theory and complex geometry, Grundlehren Math. Wiss. 289 (Springer-Verlag, Berlin, Heidelberg, New York, 1988).

[89] Yu.I. Manin, Quantum groups and non-commutative differential geometry, Montréal University preprint CRM-1561, 1988.

[90] Yu.I. Manin, Topics in noncommutative geometry (Princeton University Press, Princeton, NJ, 1991).

[91] J.L. Martin, "Generalized classical dynamics, and the "classical analogue" of a Fermi oscillator', Proc. Roy. Soc. London Ser. A 251 (1959), 536-543.

[92] S. Matsumoto and K. Kakazu, 'A note on topology of supermanifolds', J. Math. Phys. 27 (1986), 2960-2962.

[93] E. Michael, 'Locally multiplicatively-convex topological algebras', Mem. Amer. Math. Soc. 11 (1952).

[94] I. Moerdijk and G.E. Reyes, Models for smooth infinitesimal analysis (Springer-Verlag, Berlin, Heidelberg, New York, 1991).

[95] V. Molotkov, Infinite dimensional $\mathbb{Z}_{2}^{k}$-supermanifolds (ICTP preprint IC/84/183, Trieste, 1984).

[96] J. Monterde and A. Montesinos, 'Integral curves of derivations', Ann. Global Anal. Geom. 6 (1988), 177-189.

[97] P. Nieuwenhuizen, 'Supergravity', Phys. Rep. 68 (1981), 189-398.

[98] F. Ongay-Larios and O.A. Sánchez-Valenzuela, ' $\mathbf{R}^{1 / 1}$-supergroup actions and superdifferential equations', Proc. Amer. Math. Soc. 116 (1992), 843-850.

[99] I. Penkov, 'Classical Lie supergroups and their superalgebras and their representations', Publ. Inst. Fourier 117 (1988).

[100] V. Pestov, 'On a "super" version of Lie's third fundamental theorem', Lett. Math. Phys. 18 (1989), 27-33.

[101] V. Pestov, 'Interpreting superanalyticity in terms of convergent series', Classical Quantum Gravity 6 (1989), L145-L159.

[102] V. Pestov, 'Even sectors of Lie superalgebras as locally convex Lie algebras', J. Math. Phys. 32 (1991), 24-32.

[103] V. Pestov, 'Ground algebras for superanalysis', Rep. Math. Phys. 29 (1991), 275-287.

[104] V.G. Pestov, 'Nonstandard hulls of normed Grassmann algebras and their application in superanalysis', Soviet Math. Dokl. 43 (1991), 456-460.

[105] V.G. Pestov, 'A contribution to nonstandard superanalysis', J. Math. Phys. 33 (1992), 3263-3273.

[106] V.G. Pestov, 'General construction of Banach-Grassmann algebras', Atti Accad. Naz. Lincei Rend. (9) 3 (1992), 223-231. 
[107] V.G. Pestov, 'Universal arrows to forgetful functors from categories of topological algebra', Bull. Austral. Math. Soc. 48 (1993), 209-249.

[108] V.G. Pestov, 'Soul expansion of $G^{\infty}$ superfunctions', J. Math. Phys. 34 (1993), 3316-3323.

[109] V.G. Pestov, 'An analytic structure emerging in the presence of infinitely many odd coordinates', New Zealand J. Math. 22 (1993), 75-84.

[110] V.G. Pestov, 'On enlargability of infinite-dimensional Lie superalgebras', J. Geom. Phys. 10 (1993), 295-314.

[111] V.G. Pestov, 'A correspondence between finite-dimensional Lie superalgebras and supergroups', Extracta Mathematicae 7 (1992), 119-125.

[112] V.G. Pestov, On even collective effects in purely odd superspaces, Research Report RP-93-132, Dept. Math., Victoria University of Wellington, October 1993.

[113] P.F. Picken, 'Supergroups have no extra topology', J. Phys. A 16 (1983), 3457-3462.

[114] J. Rabin, 'Supermanifold cohomology and the Wess-Zumino term of the covariant superstring action', Comm. Math. Phys. 108 (1987), 375-389.

[115] J. Rabin and L. Crane, 'Global properties of supermanifolds', Comm. Math. Phys. 100 (1985), 141-160.

[116] J. Rabin and L. Crane, 'How different are the supermanifolds of Rogers and DeWitt?', Comm. Math. Phys. 102 (1985), 123-137.

[117] T. Regge, 'The group manifold approach to unified gravity', in Relativité, groupes et topologie II (Elsevier Sci. Publishers, Amsterdam, 1984), pp. 933-1006.

[118] V. Rittenberg and M. Scheunert, 'Elementary construction of graded Lie groups', J. Math. Phys. 19 (1978), 709-713.

[119] A. Robinson, Non-Standard Analysis (North-Holland Publ. Co, Amsterdam, London, 1970).

[120] A. Rogers, 'A global theory of supermanifolds', J. Math. Phys. 21 (1980), 1352-1365.

[121] A. Rogers, 'Some examples of compact supermanifolds with non-Abelian fundamental group', J. Math. Phys. 22 (1981), 443-444.

[122] A. Rogers, 'Super Lie groups: global topology and local structure', J. Math. Phys. 22 (1981), 939-945.

[123] A. Rogers, 'Aspects of the geometrical approach to supermanifolds', in Mathematical Aspects of Superspace, (H.-J. Seifert et al, Editors) (D. Reidel Publ. Co., 1984), pp. 135-147.

[124] A. Rogers, 'Integration on supermanifolds', in Mathematical Aspects of Superspace, (H.-J. Seifert et al, Editors) (D. Reidel Publ. Co., 1984), pp. 149-160.

[125] A. Rogers, 'Graded manifolds, supermanifolds and infinite-dimensional Grassmann algebras', Comm. Math. Phys. 105 (1986), 375-384.

[126] A.A. Rosly, A.S. Schwarz and A.A. Voronov, 'Superconformal geometry and string theory', Comm. Math. Phys. 120 (1989), 437-450.

[127] G.-C. Rota and J.A. Stein, 'Supersymmetric Hilbert space', Proc. Nat. Acad. Sci. USA 87 (1990), 2521-2524.

[128] M.J. Rothstein, Supermanifolds over an arbitrary graded commutative algebra, Ph.D. the- 
sis (UCLA, Los Angeles, CA, 1984).

[129] M.J. Rothstein, 'The axioms of supermanifolds and a new structure arising from them', Trans. Amer. Math. Soc. 297 (1986), 159-180.

[130] M.J. Rothstein, 'Equivariant splittings of supermanifolds', J. Geom. Phys. 12 (1993), 145-152.

[131] A. Salam and J. Strathdee, 'Super-gauge transformations', Nuclear Phys. B 76 (1974), 477-482.

[132] O.A. Sánchez Valenzuela, 'Linear supergroup actions. I: On the defining properties', Trans. Amer. Math. Soc. 307 (1988), 569-595.

[133] O.A. Sánchez Valenzuela, 'Matrix computations in linear superalgebra', Linear Algebra Appl. 111 (1988), 151-181.

[134] O.A. Sánchez Valenzuela, Differential-geometric approach to supervector bundles, CIMATpreprint 1991, Centro de Investigación en Matemáticas, Guanajuato, Mexico (1991).

[135] O.A. Sánchez Valenzuela, 'A note on integration of 1-superforms along $(1,1)$-dimensional lines', in Proc. XIX Int. Conf. on Group Theor. Methods in Physics (Salamanca, 1992), Anales de Física de la Real Soc. Esp. de Fís. Vol. II, 1993, pp. 277-280.

[136] Th. Schmitt, Infinite-dimensional supermanifolds $I$, Report R-Math-08/88 (Akad. Wiss. DDR, Berlin, 1988).

[137] A.S. Schwarz, 'Supergravity, complex geometry and G-structures', Comm. Math. Phys. $87(1982 / 83), 37-63$.

[138] J. Schwinger, 'A note on the quantum dynamical principle', Philos. Mag. 44 (1953), 1171-1193.

[139] K.D. Stroyan and W.A.J. Luxemburg, Introduction to the theory of infinitesimals (Academic Press, New York, 1976).

[140] G. Takeuti, 'Boolean valued analysis', in Lecture Notes in Math. 753 (Springer-Verlag, New York, 1979), pp. 714-731.

[141] P. Teofilatto, 'Enlargeable graded Lie algebras of supersymmetry', J. Math. Phys. 28 (1987), 991-996.

[142] V.S. Vladimirov and I.V. Volovich, 'Superanalysis - I. Differential calculus', Theoret. and Math. Phys. 59 (1984), 317-335.

[143] V.S. Vladimirov and I.V. Volovich, 'Superanalysis - II. Integral calculus', Theoret. and Math. Phys. 60 (1984), 743-765.

[144] D.V. Volkov and V.P. Akulov, 'Is the neutrino a Goldstone particle?', Phys. Lett. B 46 (1973), 109-110.

[145] J. Wess and B. Zumino, 'Supergauge transformations in four dimensions', Nuclear Phys. B 70 (1974), 39-50.

[146] E. Witten, 'Supersymmetry and Morse theory', J. Differential Geom. 17 (1982), 661-692.

[147] D.N. Yetter, 'Models for synthetic supergeometry', Cahiers de topologie et géometrie différentielle catégoriques 29 (1988), 87-108. 
Department of Mathematics

Victoria University of Wellington

Wellington

New Zealand

e-mail: vladimir.pestov@vuw.ac.nz 